\title{
Toxicity profile of epidermal growth factor receptor tyrosine kinase inhibitors in patients with epidermal growth factor receptor gene mutation-positive lung cancer (Review)
}

\author{
MASAYUKI TAKEDA and KAZUHIKO NAKAGAWA \\ Department of Medical Oncology, Kinki University Faculty of Medicine, Osaka-Sayama, Osaka 589-8511, Japan
}

Received June 4, 2016; Accepted August 18, 2016

DOI: $10.3892 /$ mco.2016.1099

\begin{abstract}
Recent progress in the research on the molecular biology of lung cancer revealed that the clinical response to epidermal growth factor receptor (EGFR) tyrosine kinase inhibitors (TKIs) is associated with the presence of activating EGFR mutations. Three EGFR-TKIs, namely afatinib, erlotinib and gefitinib, are currently available for the treatment of patients with EGFR mutation-positive non-small-cell lung cancer (NSCLC). Due to the dearth of published phase III trials prospectively evaluating the effects of one EGFR-TKI in comparison with another in such patients, the decision-making regarding which agent to recommend to any given patient lies with the treating physician. Given the potential long-term exposure of such patients to EGFR-TKIs, the toxicological properties of these drugs in such patients may differ from those observed in unselected patients. The aim of the present study was to provide an overview of the key adverse events (rash, diarrhea, hepatotoxicity and interstitial lung disease) reported for EGFR-TKIs in clinical trials including patients with advanced NSCLC.
\end{abstract}

\section{Contents}

1. Introduction

2. Pooled safety data from prospective trials for $E G F R$ mutation-positive NSCLC

3. Afatinib vs. gefitinib (LUX-Lung 7)

4. Erlotinib vs. gefitinib (WJOG 5108L trial)

5. Mechanisms underlying safety differences among EGFR-TKIs

6. Conclusions

Correspondence to: Dr Masayuki Takeda, Department of Medical Oncology, Kinki University Faculty of Medicine, 377-2 Ohno-higashi, Osaka-Sayama, Osaka 589-8511, Japan

E-mail: takedamasa2004@yahoo.co.jp

Key words: non-small-cell lung cancer, epidermal growth factor receptor, adverse event, gefitinib, erlotinib, afatinib

\section{Introduction}

Recent progress in the research on the molecular biology of lung cancer has led to the identification of oncogenic drivers, such as mutation of the epidermal growth factor receptor (EGFR) gene and translocation of the anaplastic lymphoma kinase (ALK) gene (1-5). The discovery that activating mutations of EGFR are associated with a marked response to the EGFR tyrosine kinase inhibitor (TKI) gefitinib in patients with non-small-cell lung cancer (NSCLC) led to the undertaking of several randomized phase III studies to compare EGFR-TKIs with platinum doublets in patients with EGFR mutation-positive NSCLC (6-11). The results of these trials confirmed the superiority of EGFR-TKI treatment over standard chemotherapy in terms of progression-free survival (PFS) in these biomarker-selected patients. Three EGFR-TKIs, namely gefitinib, erlotinib and afatinib, are currently available for the first-line therapy of patients with advanced NSCLC positive for $E G F R$ mutations. However, there have been no published phase III trials that have prospectively evaluated the effects of one EGFR-TKI in comparison with another in such patients. The decision to recommend to a patient one EGFR-TKI over another therefore lies with the treating physician in clinical practice. Differences in safety among these three EGFR-TKIs have been identified and may affect treatment-related decisions. Given the potential long-term exposure of biomarker-selected patients to EGFR-TKIs, however, the toxicological properties of these agents in such patients may differ from those observed in unselected patients. The aim of this review was to provide an overview of key adverse events, such as rash, diarrhea, hepatotoxicity and interstitial lung disease (ILD), reported for EGFR-TKIs in clinical trials that were largely restricted to patients with EGFR mutation-positive tumors.

2. Pooled safety data from prospective trials for $E G F R$ mutation-positive NSCLC

To the best of our knowledge, no randomized phase II or III trials performing a prospective evaluation of the efficacy and safety of one EGFR-TKI in comparison with another in patients with EGFR mutation-positive NSCLC had been fully published by early 2015 . Therefore, a pooled safety analysis of the incidence of severe (grade $\geq 3$ ) toxicity according to the type of EGFR-TKI administered was undertaken, using data 
Table I. Frequency (\%) of grade $\geq 3$ adverse events according to the type of EGFR-TKI.

\begin{tabular}{|c|c|c|c|c|}
\hline \multirow[b]{2}{*}{ Adverse event } & \multicolumn{2}{|c|}{$\begin{array}{c}\text { WJOG 5108L } \\
\text { (72\% EGFR mutation-positive) }\end{array}$} & \multicolumn{2}{|c|}{$\begin{array}{c}\text { LUX-Lung } 7 \\
\text { (all EGFR mutation-positive) }\end{array}$} \\
\hline & Erlotinib & Gefitinib & Gefitinib & Afatinib \\
\hline Hepatotoxicity $^{\mathrm{a}}$ & $3.3 / 2.2$ & $13.0 / 6.1$ & $8.2 / 2.5$ & $0 / 0$ \\
\hline Rash & 18.1 & 2.2 & 3.1 & 9.4 \\
\hline Diarrhea & 3.3 & 2.2 & 1.3 & 12.5 \\
\hline ILD & 1.4 & 0.4 & 2.5 (all grades) & 0 \\
\hline
\end{tabular}

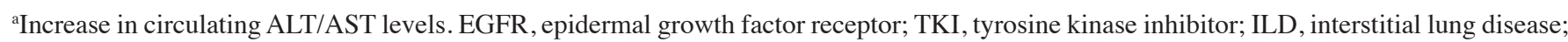
ALT, alanine aminotransferase; AST, aspartate aminotransferase.

extracted from prospective clinical trials including advanced NSCLC patients harboring EGFR mutations (12). A search through the PubMed database identified 21 phase II or III trials of EGFR-TKIs including such patients. The incidence of grade $\geq 3$ liver toxicity was significantly higher in gefitinib-treated patients compared with that in patients treated with erlotinib (18 vs. 5.4\%, respectively), as well as in erlotinib-treated patients compared with that in patients treated with afatinib (5.4 vs. 1.7\%, respectively). The frequency of grade $\geq 3$ rash was higher among afatinib-treated patients compared with that among those treated with erlotinib (15 vs. $8.8 \%$, respectively), as well as among erlotinib-treated patients compared with those treated with gefitinib $(8.8$ vs. $3.5 \%$, respectively). Grade $\geq 3$ diarrhea occurred more frequently in afatinib-treated patients compared with those treated with erlotinib (9.6 vs. $2.7 \%$, respectively), whereas the frequency of this toxicity did not differ significantly between patients treated with erlotinib and those receiving gefitinib ( 2.7 vs. $1.1 \%$, respectively). Although ILD is rare, it is potentially fatal; thus, we also assessed the frequency of this toxicity at grade $\geq 3$ and found that its incidence did not differ significantly among the three groups of patients $(2.2 \%$ for gefitinib vs. $0.6 \%$ for erlotinib or afatinib). Therefore, there were clinically significant differences in the occurrence of hepatotoxicity, rash and diarrhea among patients treated with these three EGFR-TKIs, although such differences have been directly investigated in only a limited number of patients to date.

\section{Afatinib vs. gefitinib (LUX-Lung 7)}

Early results have been reported for a randomized, open-label phase IIb trial of afatinib vs. gefitinib as first-line treatment for patients with advanced lung adenocarcinoma who were positive for exon 19 deletions or the L858R point mutation of EGFR (LUX-Lung 7, ClinicalTrials.gov identifier NCT 01466660) (13). The primary endpoint of this study was to compare afatinib with gefitinib in terms of PFS, overall survival (OS) and time to treatment failure. A total of 571 patients were screened, 319 of whom were randomized to either the afatinib $(n=160)$ or gefitinib $(n=159)$ arms. Afatinib treatment was associated with a significantly improved PFS [median, 11.0 vs. 10.9 months; hazard ratio $(\mathrm{HR})=0.73$,
$\mathrm{P}=0.0165]$, time to treatment failure (median, $13.7 \mathrm{vs}$. 11.5 months; $\mathrm{HR}=0.73, \mathrm{P}=0.0073)$ and objective response rate (70 vs. 56\%, $\mathrm{P}=0.0083$ ) compared with gefitinib. Treatment with either drug was generally tolerable, resulting in equally low rates of treatment-related discontinuation in the two arms $(6.3 \%)$. The frequency of grade $\geq 3$ diarrhea (12.5 vs. $1.3 \%$ ) and rash or acne (9.4 vs. $3.1 \%$ ) was higher in the afatinib arm compared with that in the gefitinib arm, whereas the frequency of a grade $\geq 3$ increase in the circulating levels of alanine aminotransferase (ALT)/aspartate aminotransferase (AST) was higher with gefitinib compared with afatinib (8.2/2.5 vs. 0/0\%) (Table I). Drug-related ILD was reported in 4 patients on gefitinib and none on afatinib. These results are thus consistent with those of our pooled analysis, demonstrating that grade $\geq 3$ rash or diarrhea occur more frequently among patients treated with afatinib, whereas hepatotoxicity is more frequent among those treated with gefitinib.

\section{Erlotinib vs. gefitinib (WJOG 5108L trial)}

A multicenter, randomized phase III study was designed to demonstrate the non-inferiority of gefitinib to erlotinib for advanced lung adenocarcinoma (WJOG 5108L, UMIN000002014) (14). Previously treated patients were randomized in a $1: 1$ ratio to receive either erlotinib $(150 \mathrm{mg}$ daily) or gefitinib (250 mg daily) according to gender, disease stage, EGFR mutation status, performance status, smoking history, line of chemotherapy and institution. The protocol was amended in December, 2011, as the Pharmaceuticals and Medical Devices Agency of Japan limited the use of gefitinib to patients harboring EGFR mutations. A total of 561 patients were accrued, 2 of whom proved to be ineligible; finally, 280 and 279 subjects were randomly assigned to the erlotinib and gefitinib arms, respectively, including 198 (70.7\% in the erlotinib arm) and 203 (72.8\% in the gefitinib arm) with tumors positive for activating mutations of EGFR. The median PFS was 6.5 and 7.5 months $(\mathrm{HR}=1.125, \mathrm{P}=0.257)$ and the median OS was 22.8 and 24.5 months $(\mathrm{HR}=1.038, \mathrm{P}=0.768)$ for gefitinib and erlotinib, respectively. The median PFS in patients harboring EGFR mutations was 8.3 and 10.0 months $(\mathrm{HR}=1.093, \mathrm{P}=0.424)$ for gefitinib and erlotinib, respectively. The study did not demonstrate non-inferiority of gefitinib compared with erlotinib in terms of PFS in patients with 
lung adenocarcinoma according to the predefined criteria. The incidence of grade $\geq 3$ rash was higher in the erlotinib arm compared with that in the gefitinib arm (18.1 vs. $2.2 \%$, respectively), whereas that of grade $\geq 3$ diarrhea did not differ significantly between the erlotinib and gefitinib arms (3.3 vs. $2.2 \%$, respectively) (Table I). A grade $\geq 3$ increase in ALT/AST was more frequent with gefitinib compared with erlotinib (13.0/6.1 vs. 3.3/2.2\%, respectively). A $4 \%$ incidence of ILD of any grade was reported in each group, with 3 patients in the erlotinib arm experiencing grade 5 ILD. Thus, there was a significant difference in the frequencies of rash and hepatotoxicity between the two treatment arms, although the toxicity profile for this trial may not accurately represent that for a homogeneous EGFR mutation-positive population, given that only $72 \%$ of the trial patients harbored such mutations.

\section{Mechanisms underlying safety differences among EGFR-TKIs}

EGFR-TKI-induced rash and diarrhea are usually mild to moderate in severity. The frequency of these adverse events at grade $\geq 3$ appears to be higher among patients treated with afatinib compared with those treated with gefitinib. These differences may be explained in part by the mechanism of drug action. EGFR is expressed in epithelia and helps to maintain mucosal integrity and to promote mucosal repair in the gut, as well as to maintain the protective barrier of the skin. Afatinib exhibits a higher affinity for the kinase domain of EGFR compared with gefitinib, and the irreversible tyrosine kinase blockade mediated by afatinib may result in more sustained suppression of EGFR signaling compared with that induced by the reversible inhibitor gefitinib (15). However, the frequency of grade $\geq 3$ rash appears to be higher among patients treated with erlotinib compared with those treated with gefitinib, with this difference possibly being attributable to a difference in pharmacological properties between the two drugs. Administration of erlotinib at the maximum tolerated and approved dose of $150 \mathrm{mg}$ once daily thus gives rise to a steady-state plasma trough concentration that is $\sim 3.5$ times that for gefitinib administered at the recommended dose ( one-third of the maximum tolerated dose) of $250 \mathrm{mg}$ once daily $(16,17)$.

The reason for liver dysfunction being more frequent among patients treated with gefitinib compared with those receiving other EGFR-TKIs remains unclear. A previous study evaluated the frequency of adverse events according to the functional status of CYP2D6, an enzyme that mediates the metabolism of gefitinib, in unselected patients treated with gefitinib or erlotinib (18). That study demonstrated that reduced CYP2D6 function was associated with an increased risk of rash, but not of liver dysfunction, in the gefitinib cohort, suggesting that the hepatotoxicity of gefitinib may not be dose-dependent. Another study investigated the association of polymorphisms of the CYP2D6 gene with gefitinib hepatotoxicity and found that gene variants associated with reduced CYP2D6 activity were not predictive of hepatotoxicity (19). A case series study demonstrated that erlotinib was effective and well-tolerated as a treatment option following discontinuation of gefitinib as a result of drug-related hepatotoxicity (20). Erlotinib and gefitinib share a 4-anilinoquinazoline base structure, but they differ in the substituents attached to the quinazoline and anilino rings. Minor differences in the chemical structures of these compounds may thus affect hepatotoxicity.

\section{Conclusions}

The application of the EGFR-TKIs gefitinib, erlotinib and afatinib have improved the clinical outcome of patients with EGFR mutation-positive NSCLC, but these agents are associated with a number of serious adverse events that require management. The safety profile of different EGFR-TKIs should be taken into consideration by the treating physician when selecting the most appropriate drug with regard to mitigation of the risk for certain types of toxicity.

\section{Acknowledgements}

The present study was supported in part by the Japan Society for the Promotion of Science-KAKENHI (grant no. 15K21525).

\section{References}

1. Lynch TJ, Bell DW, Sordella R, Gurubhagavatula S, Okimoto RA, Brannigan BW, Harris PL, Haserlat SM, Supko JG, Haluska FG, et al: Activating mutations in the epidermal growth factor receptor underlying responsiveness of non-small-cell lung cancer to gefitinib. N Engl J Med 350: 2129-2139, 2004

2. Paez JG, Jänne PA, Lee JC, Tracy S, Greulich H, Gabriel S, Herman P, Kaye FJ, Lindeman N, Boggon TJ, et al: EGFR mutations in lung cancer: Correlation with clinical response to gefitinib therapy. Science 304: 1497-1500, 2004.

3. Pao W, Miller V, Zakowski M, Doherty J, Politi K, Sarkaria I, Singh B, Heelan R, Rusch V, Fulton L, et al: EGF receptor gene mutations are common in lung cancers from 'never smokers' and are associated with sensitivity of tumors to gefitinib and erlotinib. Proc Natl Acad Sci USA 101: 13306-13311, 2004.

4. Soda M, Choi YL, Enomoto M, Takada S, Yamashita Y, Ishikawa S, Fujiwara S, Watanabe H, Kurashina K, Hatanaka H, et al: Identification of the transforming EML4-ALK fusion gene in non-small-cell lung cancer. Nature 448: 561-566, 2007.

5. Takeda M, Okamoto I, Sakai K, Kawakami H, Nishio K and Nakagawa K: Clinical outcome for EML4-ALK-positive patients with advanced non-small-cell lung cancer treated with first-line platinum-based chemotherapy. Ann Oncol 23: 2931-2936, 2012.

6. Mitsudomi T, Morita S, Yatabe Y, Negoro S, Okamoto I, Tsurutani J, Seto T, Satouchi M, Tada H, Hirashima T, et al: Gefitinib versus cisplatin plus docetaxel in patients with non-small-cell lung cancer harbouring mutations of the epidermal growth factor receptor (WJTOG3405): An open label, randomised phase 3 trial. Lancet Oncol 11: 121-128, 2010.

7. Maemondo M, Inoue A, Kobayashi K, Sugawara S, Oizumi S, Isobe $\mathrm{H}$, Gemma A, Harada M, Yoshizawa H, Kinoshita I, et al: Gefitinib or chemotherapy for non-small-cell lung cancer with mutated EGFR. N Engl J Med 362: 2380-2388, 2010.

8. Zhou C, Wu YL, Chen G, Feng J, Liu XQ, Wang C, Zhang S, Wang J, Zhou S, Ren S, et al: Erlotinib versus chemotherapy as first-line treatment for patients with advanced EGFR mutation-positive non-small-cell lung cancer (OPTIMAL, CTONG-0802): A multicentre, open-label, randomised, phase 3 study. Lancet Oncol 12: 735-742, 2011.

9. Rosell R, Carcereny E, Gervais R, Vergnenegre A, Massuti B, Felip E, Palmero R, Garcia-Gomez R, Pallares C, Sanchez JM, et al: Erlotinib versus standard chemotherapy as first-line treatment for European patients with advanced EGFR mutation-positive non-small-cell lung cancer (EURTAC): A multicentre, open-label, randomised phase 3 trial. Lancet Oncol 13: 239-246, 2012.

10. Sequist LV, Yang JC, Yamamoto N, O'Byrne K, Hirsh V, Mok T, Geater SL, Orlov S, Tsai CM, Boyer M, et al: Phase III study of afatinib or cisplatin plus pemetrexed in patients with metastatic lung adenocarcinoma with EGFR mutations. J Clin Oncol 31: 3327-3334, 2013 
11. Wu YL, Zhou C, Hu CP, Feng J, Lu S, Huang Y, Li W, Hou M, Shi JH, Lee KY, et al: Afatinib versus cisplatin plus gemcitabine for first-line treatment of Asian patients with advanced non-small-cell lung cancer harbouring EGFR mutations (LUX-Lung 6): An open-label, randomised phase 3 trial. Lancet Oncol 15: 213-222, 2014.

12. Takeda M, Okamoto I and Nakagawa K: Pooled safety analysis of EGFR-TKI treatment for EGFR mutation-positive non-small cell lung cancer. Lung Cancer 88: 74-79, 2015.

13. Park K, Tan E, Zhang L, Hirsh V, O'Byrne K, Boyer M, Yang JC, Mok T, Kim M, Massey D, et al: Afatinib vs gefitinib as first-line treatment for patients with advanced non-small cell lung cancer harboring activating EGFR mutations: Results of the global, randomized, open-label, Phase IIb trial LUX-Lung 7. Ann Oncol 26 (Suppl 9): ix161-ix162, 2015.

14. Urata Y, Katakami N, Morita S, Kaji R, Yoshioka H, Seto T, Satouchi M, Iwamoto Y, Kanehara M, Fujimoto D, et al: Randomized phase III study comparing gefitinib with erlotinib in patients with previously treated advanced lung adenocarcinoma: WJOG 5108L. J Clin Oncol pii: JCO634154, 2016.

15. Nelson V, Ziehr J, Agulnik M and Johnson M: Afatinib: Emerging next-generation tyrosine kinase inhibitor for NSCLC. Onco Targets Ther 6: 135-143, 2013.
16. Li J, Karlsson MO, Brahmer J, Spitz A, Zhao M, Hidalgo M and Baker SD: CYP3A phenotyping approach to predict systemic exposure to EGFR tyrosine kinase inhibitors. J Natl Cancer Inst 98: 1714-1723, 2006.

17. Tan AR, Yang X, Hewitt SM, Berman A, Lepper ER, Sparreboom A, Parr AL, Figg WD, Chow C, Steinberg SM, et al: Evaluation of biologic end points and pharmacokinetics in patients with metastatic breast cancer after treatment with erlotinib, an epidermal growth factor receptor tyrosine kinase inhibitor. J Clin Oncol 22: 3080-3090, 2004.

18. Suzumura T, Kimura T, Kudoh S, Umekawa K, Nagata M, Matsuura K, Tanaka H, Mitsuoka S, Yoshimura N, Kira Y, et al: Reduced CYP2D6 function is associated with gefitinib-induced rash in patients with non-small cell lung cancer. BMC Cancer 12: 568, 2012.

19. Takimoto T, Kijima T, Otani Y, Nonen S, Namba Y, Mori M, Yokota S, Minami S, Komuta K, Uchida J, et al: Polymorphisms of CYP2D6 gene and gefitinib-induced hepatotoxicity. Clin Lung Cancer 14: 502-507, 2013.

20. Takeda M, Okamoto I, Tsurutani J, Oiso N, Kawada A and Nakagawa K: Clinical impact of switching to a second EGFR-TKI after a severe AE related to a first EGFR-TKI in EGFR-mutated NSCLC. Jpn J Clin Oncol 42: 528-533, 2012. 